\title{
Effect of Propofol on Human Neutrophil Apoptosis in vitro
}

\author{
Kishikawa $H^{*}$, Kawaguchi H, Tsuboko Y, Nakatani H, Ishikawa M \\ and Sakamoto A \\ Department of Anesthesiology, Nippon Medical School, Tokyo, Japan
}

*Corresponding author: Hiroaki Kishikawa, Department of Anesthesiology, Nippon

\author{
Research Article \\ Volume 2 Issue 1
}

Received Date: February 27, 2017

Published Date: March 24, 2017

Medical School, 1-1-5 Sendagi, Bunkyo-ku, Tokyo, Japan Tel: +81-3-3822-2131; E-mail: h-k@nms.ac.jp

\section{Abstract}

Purpose: Propofol is clinically used for anesthesia and sedation. It remains unclear whether propofol induces human neutrophil apoptosis. We sought to determine the effect of propofol on neutrophil apoptosis in vitro.

Methods: Peripheral neutrophils from healthy volunteers were treated with different concentrations of propofol $(0,5$, and $50 \mu \mathrm{g} / \mathrm{ml}$ ). Apoptosis and the mitochondrial trans membrane potential (MTP) were detected by fluorescence microscopy after Hoechst 33342 and JC-1 staining. Caspase activity was measured using luminescent substrates.

Results: A high concentration $(50 \mu \mathrm{g} / \mathrm{ml})$ of propofol significantly increased apoptosis, reduced MTP, and enhanced caspase- 9 and caspase-3/7 activities. The addition of an MTP stabilizer and caspase-9 and -3 inhibitors decreased propofol-induced apoptosis. Caspase-8 activity was not affected by $50 \mu \mathrm{g} / \mathrm{ml}$ of propofol and propofol-induced apoptosis was not suppressed by the caspase- 8 inhibitor. A clinically relevant concentration ( $5 \mu \mathrm{g} / \mathrm{ml})$ of propofol did not affect apoptosis rate, MTP and caspase activities.

Conclusion: Propofol at the clinical relevant concentrations had no effect on neutrophil apoptosis, although a high dose of propofol increased rate of apoptosis. The mechanism of propofol-induced apoptosis is involved with the mitochondrial pathway.

\section{Introduction}

Neutrophils are cardinal cellular effectors of the innate host response to infection and injury. Neutrophils spontaneously die by apoptosis within approximately 1-2 days under normal conditions [1-3]. Delayed neutrophil apoptosis is associated with organ injury during sepsis $[4,5]$, by contrast, accelerated apoptosis contributes to the immunodeficiency in acquired immunodeficiency syndrome [6,7]. Thus, the rate of neutrophil apoptosis is critical for efforts to resolve inflammations.
Propofol is an intravenous anesthetic agent widely used for the induction and maintenance of anesthesia and for sedation in intensive care units (ICUs) [8]. Although several reports have indicated that propofol anesthesia reduces the postoperative counts of neutrophils $[9,10]$ and higher concentrations of propofol have pro-apoptotic effects on other leukocytic cells in vitro $[11,12]$, the effect of propofol on neutrophil apoptosis remains unclear. If propofol has an anti or pro-apoptotic effect on neutrophil, it could influence the resolutions of inflammation or immune dysfunction. 


\section{Anaesthesia \& Critical Care Medicine Journal}

The aims of this study were to evaluate whether propofol induces human peripheral neutrophil apoptosis in vitro and to confirm the changes in mitochondrial transmembrane potential (MTP) and the activity of caspases by propofol.

\section{Materials and Methods}

\section{Human Peripheral Neutrophil Isolation and Culture}

After obtaining approval from the Institutional Ethics Committee, informed consent was obtained from each healthy participant prior to the start of the study. Human heparinized peripheral venous blood was overlaid onto a Mono-Poly resolving medium (Dainippon Sumitomo Pharma, Osaka, Japan) and peripheral neutrophils were isolated by density gradient centrifugation [13]. The purity of neutrophils was assessed microscopically and viability was confirmed to be above $95 \%$ using the try pan blue exclusion test. Isolated neutrophils were cultured at a concentration of $1 \times 10^{6}$ cells $/ \mathrm{ml}$ in 24 -well plates with RPMI 1640 medium (Sigma, Tokyo, Japan) containing $10 \%$ heat-inactivated fetal bovine serum, $1 \%$ glutamine and $1 \%$ penicillin/streptomycin solution. The cells were incubated in a humidified atmosphere of $5 \% \mathrm{CO}_{2}$ and $95 \%$ air at $37^{\circ} \mathrm{C}$. Neutrophils were treated with or without propofol (Diprivan, AstraZeneca, Osaka, Japan). To determine the mechanism underlying propofol-induced apoptosis, we preincubated neutrophils with a mitochondrial membrane stabilizer (bongkrekic acid: BA), a caspase-8 inhibitor (Z-IETD-FMK), a caspase-9 inhibitor (Z-LEHD-FMK), or a caspase-3 inhibitor (Z-DVED-FMK) (Merck Chemicals, Tokyo, Japan) before treatment with propofol.

\section{Detection and Quantification of Nneutrophil Apoptosis}

To detect apoptosis, we stained the neutrophils with Hoechst nuclear stain as previously described $[14,15]$. After incubation, the cells were re suspended in $20 \mu \mathrm{l}$ of PBS containing $5 \mu \mathrm{g} / \mathrm{ml}$ Hoechst 33342 (Sigma) and incubated for a further $20 \mathrm{~min}$ at $37^{\circ} \mathrm{C}$. We analyzed the cells by fluorescence microscopy at $1000 \times$ magnification (BX 51; Olympus, Tokyo, Japan). Apoptosis was assessed on the basis of chromatin structure and condensation. For each condition, a minimum of 400 cells was counted and apoptosis was quantified as follows: apoptosis index (\% apoptotic cells $)=100 \times($ number of apoptotic cells $/$ total number of cells).

\section{MTP Assay}

To assess the MTP, we incubated neutrophils $(1 \times$ $10^{6} / \mathrm{ml}$ ) with $7.7 \mu \mathrm{M} \mathrm{JC}-1$ (Molecular Probes, Eugene, OR, USA) for $15 \mathrm{~min}$ at $37^{\circ} \mathrm{C}$ in the dark $[15,16]$. JC-1 fluorescence has two emission peaks, one of red fluorescence (JC-1 aggregates) indicating increased or normal MTP, and one of green fluorescence (JC-1 monomers) representing decreased MTP. After incubation with JC-1, the dye was removed and the neutrophils were analyzed by fluorescence microscopy (×1000 magnification; BX 51, Olympus). For each condition, a minimum of 400 cells were counted, and the proportion of cells with a decreased MTP was quantified as follows: neutrophils with a decreased MTP $(\%)=100 \times$ (number of cells with JC-1 monomers/total number of cells [cells with JC-1 aggregates and with JC-1 monomers]).

\section{Caspase Activity Assay}

Caspase-8, -9 and $-3 / 7$ activities were determined using the Caspase-GLO ${ }^{\mathrm{TM}}$ Assay kit (Promega, Madison, WI, USA) $[15,17]$. Caspase-GLO reagents were added directly to $1 \times 10^{4}$ neutrophils in white-walled 96-well plates, giving a final volume of $200 \mu \mathrm{l}$ per well. The contents of the wells were gently mixed and incubated at room temperature for $30 \mathrm{~min}$. We measured the luminescence of each sample in a plate-reading luminometer (GENios Plus; TECAN, Mannedorf/Zurich, Switzerland).

\section{Statistical Analysis}

All data are presented as means \pm SD. Statistical analysis was carried out using two- or one-way ANOVA followed by a post hoc Tukey-Kramer test for multiple comparisons. Differences between the two groups were analyzed by unpaired $t$-tests. Statistically significant differences were defined as those with a $P<0.05$.

\section{Results}

\section{Effects of Propofol on the Neutrophil Apoptotic Index}

Neutrophils were treated with propofol at concentrations of 5 and $50 \mu \mathrm{g} / \mathrm{ml}$; concentrations that are 1 - and 10 -fold greater than the clinically relevant plasma concentrations [11,12]. The differences between untreated neutrophils and $50 \mu \mathrm{g} / \mathrm{ml}$ propofol-treated neutrophils after Hoechst 33342 staining are shown in Figure 1a and b. After incubation for $15 \mathrm{~h}, 50 \mu \mathrm{g} / \mathrm{ml}$ propofol increased the number of apoptotic cells as 


\section{Anaesthesia \& Critical Care Medicine Journal}

compared with untreated cells. The apoptosis indices determined by Hoechst 33342 staining are shown in Figure 1c. The number of apoptotic neutrophils increased in a time dependent manner in all groups. After 15 hoursincubations, $50 \mu \mathrm{g} / \mathrm{ml}$ propofol significantly increased apoptosis index compared with other two treatments $(0$ and $5 \mu \mathrm{g} / \mathrm{ml}$ propofol). Propofol at a concentration of 5 $\mu \mathrm{g} / \mathrm{ml}$ did not significantly increase the apoptosis index compared with the untreated cells at any incubation periods

(a)

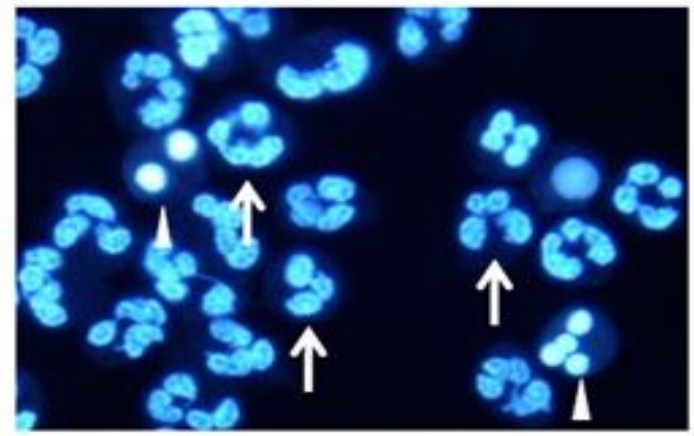

(b)

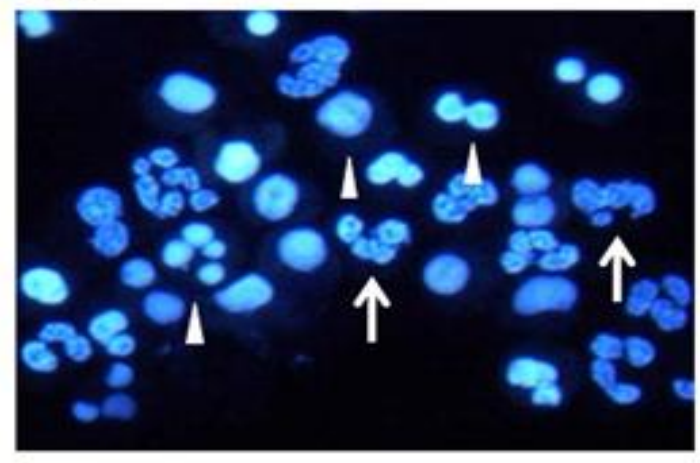

(c)

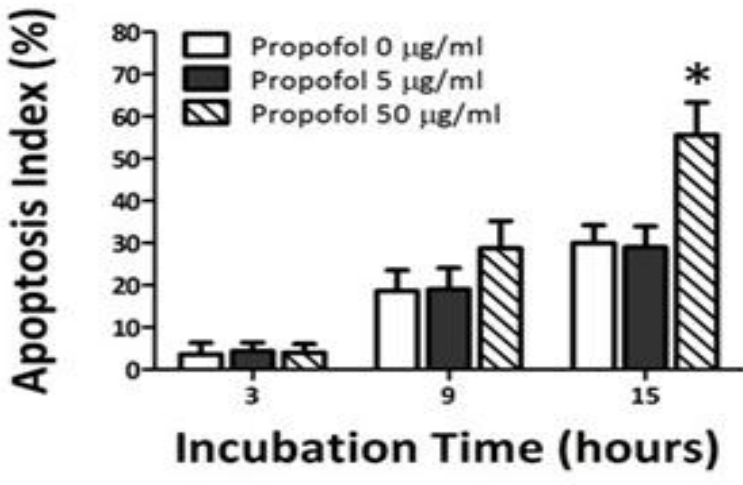

Figure 1: Effects of incubation with propofol for $15 \mathrm{~h}$ on human neutrophil apoptosis determined by Hoechst 33342 staining. Neutrophils were treated with 0 (a) or $50 \mu \mathrm{g} / \mathrm{ml}$, (b) Propofol. Representative images of cells stained with Hoechst 33342 are shown. Arrows, normal cells; arrowheads, apoptotic cells, (c) Dose-dependent effects of propofol on the apoptosis index. Data are expressed as means $\pm \mathrm{SD}(n=8) .{ }^{*} P<0.01 \mathrm{vs}$. cells treated with 0 or $5 \mu \mathrm{g} / \mathrm{ml}$ propofol.

\section{Effects of Propofol on Neutrophil MTP and Caspase Activity}

To determine the effect of propofol on the MTP and caspase activity, we treated neutrophils with $50 \mu \mathrm{g} / \mathrm{ml}$ propofol. Figure $2 \mathrm{a}$ and $\mathrm{b}$ shows the effects of 0 and 50 $\mu \mathrm{g} / \mathrm{ml}$ propofol on neutrophil MTP upon JC-1 staining. After incubation for $5 \mathrm{~h}$, neutrophils with a decreased
MTP were significantly increased in the presence of 50 $\mu \mathrm{g} / \mathrm{ml}$ propofol (Figure 2c). Although $50 \mu \mathrm{g} / \mathrm{ml}$ propofol did not affect caspase-8 activity (Fig. 3(a)), it significantly increased caspase- 9 and $-3 / 7$ activity after incubation for $8 \mathrm{~h}$ (Figure $3 \mathrm{~b} \& \mathrm{c}$ ). 


\section{Anaesthesia \& Critical Care Medicine Journal}

\section{(a)}

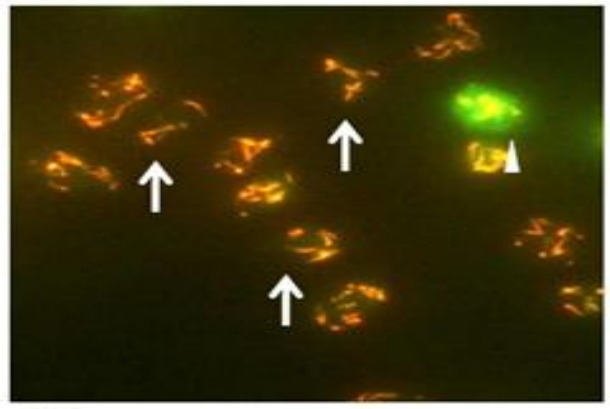

(b)

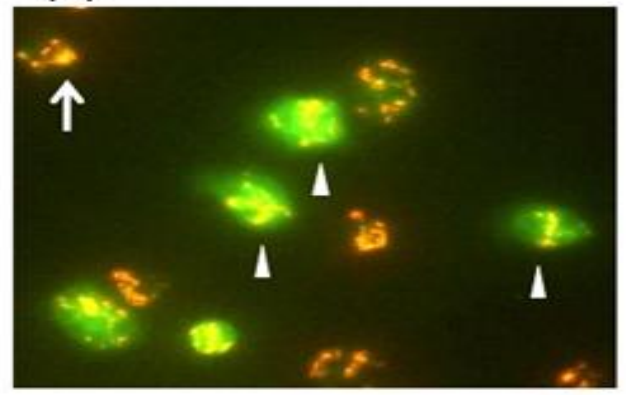

(c)

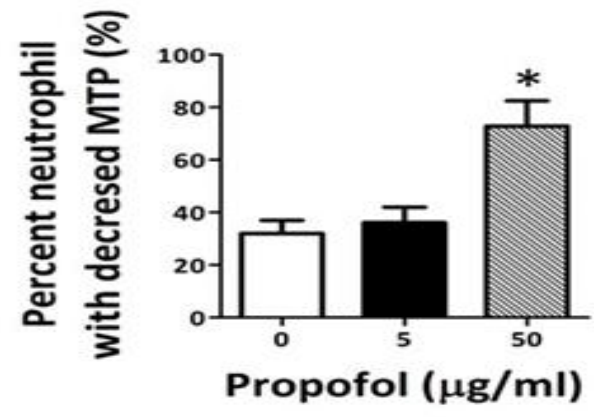

Figure 2: Effects of incubation with $50 \mu \mathrm{g} / \mathrm{ml}$ propofol for $5 \mathrm{~h}$ on neutrophil mitochondrial transmembrane potential (MTP) determined by JC-1 staining. Neutrophils were treated with 0 (a) or $50 \mu \mathrm{g} / \mathrm{ml}$ (b) propofol. Representative images of cells stained with JC-1 are shown. Arrows (red) indicate cells with JC-1 aggregates (high or normal MTP); Arrowheads (green) indicate cells with JC-1 monomers (decreased MTP). (c) Effects of propofol on the percentage of neutrophils with a decreased MTP. Data are expressed as means \pm SD $(n=8) . ~ * P<0.01$ vs. 0 or $5 \mu \mathrm{g} / \mathrm{ml}$ propofol.

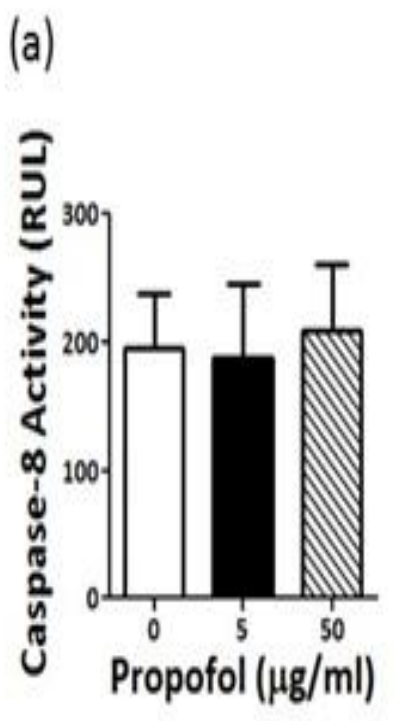

(b)

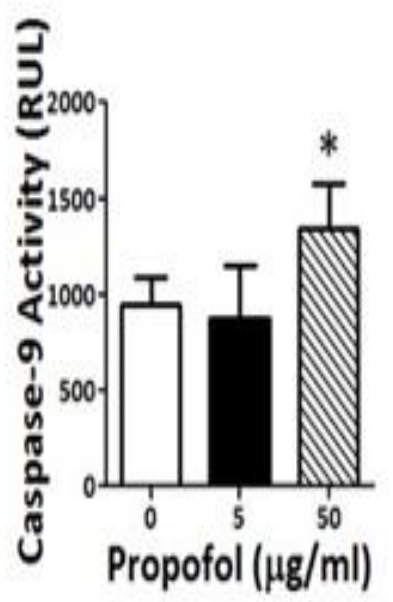

(c)

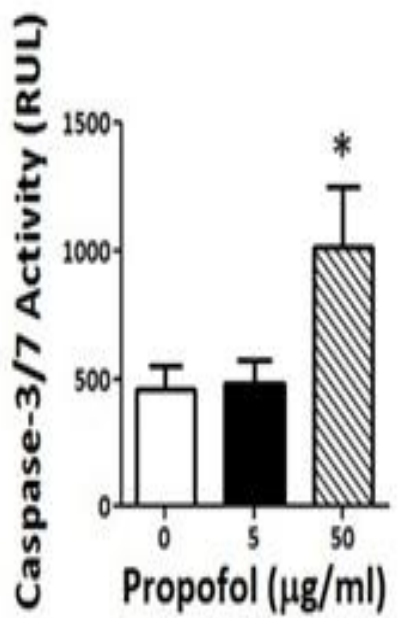

Figure 3: Effects of $50 \mu \mathrm{g} / \mathrm{ml}$ propofol on (a) caspase-8, (b) -9 and (c) $-3 / 7$ activities during neutrophil apoptosis. Activities were measured after incubation with propofol for $8 \mathrm{~h}$. Data are expressed as means $\pm \mathrm{SD}(\mathrm{n}=8)$. ${ }^{*} \mathrm{P}<0.01$ vs. 0 or $5 \mu \mathrm{g} / \mathrm{ml}$ propofol. 


\section{Anaesthesia \& Critical Care Medicine Journal}

Effects of BA and caspase inhibitors on propofolinduced neutrophil apoptosis: To evaluate the role of changes in MTP and caspase activities in apoptosis induced by $50 \mu \mathrm{g} / \mathrm{ml}$ propofol, we incubated neutrophils with $62 \mu \mathrm{M}$ BA, Z-IETD-FMK, $100 \mathrm{nM}$ Z-DVED-FMK or 100-nM Z-LEHD-FM for $1 \mathrm{~h}$ before adding $50 \mu \mathrm{g} / \mathrm{ml}$ propofol, and then determined the apoptosis index. Apoptosis induced by incubation with $50 \mu \mathrm{g} / \mathrm{ml}$ propofol for $15 \mathrm{~h}$ was significantly decreased by BA, Z-LEHD-FM and Z-DVED-FMK, but not by Z-IETD-FMK compared with the untreated cells (Figure 4).

\section{(a)}

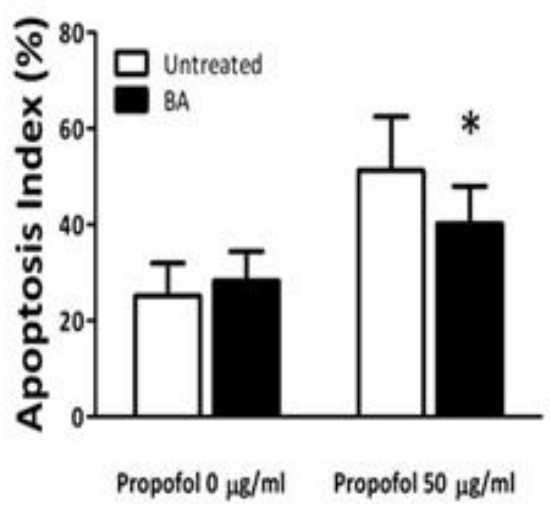

(b)

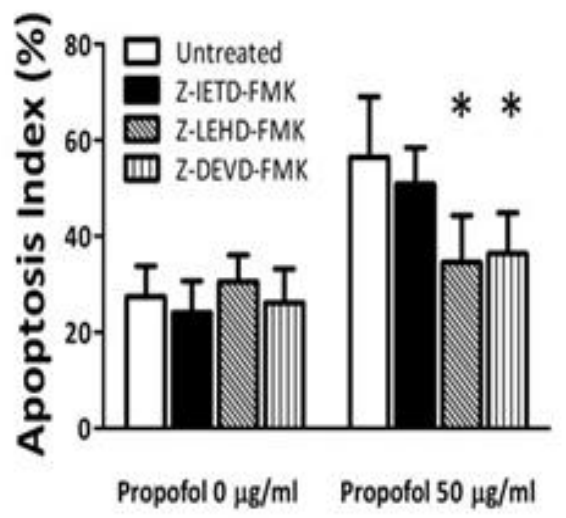

Figure 4: Effects of bongkrekic acid (BA) and caspase inhibitors on neutrophil apoptosis induced by $50 \mu \mathrm{g} / \mathrm{ml} \mathrm{propofol.}$ (a) Effects of BA on neutrophil apoptosis induced by $50 \mu \mathrm{g} / \mathrm{ml}$ propofol. Neutrophils were pre-incubated with or without $62 \mu \mathrm{M} \mathrm{BA}$ and then treated with or without $50 \mu \mathrm{g} / \mathrm{ml}$ propofol for $15 \mathrm{~h}$. Apoptosis indices were assessed by Hoechst 33342 staining after $15 \mathrm{~h}$ incubation. Data are expressed as means $\pm \mathrm{SD}(\mathrm{n}=8) .{ }^{*} \mathrm{P}<0.05 \mathrm{vs}$. cells treated with $50 \mu \mathrm{g} / \mathrm{ml}$ propofol alone. (b) Effects of inhibitors against caspase-8 (100 nMZ-IETD-FMK) caspase-9 (100 nM Z-LEHD-FMK) or caspase-3 (100 nM Z-DVED-FMK) on neutrophil apoptosis in cells treated with or without $50 \mu \mathrm{g} / \mathrm{ml}$ propofol. Neutrophils were pre-incubated with or without the indicated inhibitor. The apoptosis index was assessed by Hoechst 33342 staining after incubation for $15 \mathrm{~h}$. Data are expressed as means $\pm \mathrm{SD}(\mathrm{n}=8) .{ }^{*} \mathrm{P}<0.05$ vs. cells treated $50 \mu \mathrm{g} / \mathrm{ml}$ propofol alone.

\section{Discussion}

In this study, our results indicated that incubation of human neutrophils with a high concentration of propofol for $15 \mathrm{~h}$ increased the rate of apoptosis. On the other hand, a clinically relevant concentration of propofol did not affect rate of apoptosis after a long incubation period. We also found that apoptosis induced by $50 \mu \mathrm{g} / \mathrm{ml}$ propofol was associated with decreased MTP and the activation of caspase- 9 and $-3 / 7$ before chromatin structure and condensation of neutrophil. And our observations demonstrated that propofol-induced neutrophil apoptosis was suppressed by mitochondrial membrane stabilizer, caspase- 9 and -3 inhibitors.

Our results indicate that propofol at concentrations above those normally used in clinical settings can accelerate neutrophil apoptosis. Our finding is consistent with the reports documenting the pro-apoptotic effects of propofol on HL-60 cells, human peripheral mononuclear cells and lymphocytes [11,12]. Taken together, these findings indicate that a high concentration of propofol have a marked pro-apoptotic effect on the various human leukocytic cells.

We indicated that high doses of propofol accelerated MTP changes and caspase- 9 and 3/7 activities before chromatin condensation in neutrophils were detected. The activation of the death receptor and mitochondrial pathways during spontaneous neutrophil apoptosis has been well documented $[2,3,18]$. Caspase- 8 initiates the death receptor pathway [3], pro-apoptotic proteins released from mitochondria by reduced MTP activate caspase-9 in the mitochondrial pathway [16]. Caspase$3 / 7$ activated by caspase- 8 and -9 leads to DNA fragmentation and chromatin condensation $[2,3,18]$. Changes in the MTP and caspase activities increase in a 


\section{Anaesthesia \& Critical Care Medicine Journal}

time-dependent manner [18] and decreases of MTP and activations of caspase- 9 and $-3 / 7$ occur even before structural changes in the nucleus in this study. Therefore, our results suggest that an increase in the number of neutrophils with low MTP and activated caspase-9 and $3 / 7$ is an early hallmark of apoptosis induced by propofol.

Activation of caspase-8 is also linked to the decreased MTP in spontaneous neutrophil apoptosis [1, 3]. By contrast, we confirmed that $50 \mu \mathrm{g} / \mathrm{ml}$ propofol accelerated the decreased MTP without increase of caspase- 8 activity by propofol. In addition, apoptosis induced by propofol was depressed by mitochondrial membrane stabilizer, caspase- 9 and -3 inhibitors in this study. We speculate that the mitochondrial pathway accelerates propofol-induced apoptosis to a greater extent than the death receptor pathway.

Several limitations of this study merit discussion. First, although spontaneous neutrophil apoptosis is initiated by the death receptor and mitochondrial pathway, in our study, even in the absence of propofol, spontaneous apoptosis was not inhibited by caspase inhibitors or BA, consistent with the findings of other studies [18]. The reason why caspase inhibitors and BA did not affect apoptosis is still unclear, further studies are needed to clarify the mechanism underlying spontaneous apoptosis. Second, the present study was an in vitro basic study to evaluate the effect of propofol on neutrophil apoptosis. In the clinical setting there are many factors, surgical trauma, infection or systemic inflammation response syndrome, to influencing neutrophil apoptosis [4-7,19]. Even clinical administrations of propofol reduce the postoperative counts of neutrophils in several kinds of procedures $[9,10]$. To confirm whether the dysregulation of neutrophil apoptosis at clinically relevant doses of propofol is involved, ex vivo studies are needed in which neutrophil status can be assessed in peripheral blood samples taken from surgical or ICU patients before and after propofol administration.

In conclusion, a clinically relevant concentration of propofol had no effect on human neutrophil apoptosis from healthy volunteers in vitro, although a high dose of propofol accelerated rate of apoptosis.The mechanism of propofol-induced apoptosis is involved with the mitochondrial pathway.We believe that propofol can be safely administered to patients without accelerated inflammation or immunodeficiency due to neutrophil apoptotic dysfunction.

\section{Acknowledgments}

We are grateful to Mr. Hidemasa Takafuji and Ms. Miyuki Takatori for their assistance in this study.

\section{References}

1. Luo HR, Loison F (2008) Constitutive neutrophil apoptosis: Mechanism and regulation. Am J Hematol 83(4): 288-295.

2. Geering B, Simon HU (2011) Peculiarities of cell death mechanism in neutrophils. Cell Death Differ 18(9): 1457-1469.

3. Scheel-Toellner D, Wang K, Craddock R, Webb PR, Mc Gettrick HM, et al. (2004) Reactive oxygen species limit neutrophil life span by activating death receptor signaling. Blood 104(8): 2557-2564.

4. Cinel I, Opal SM (2009) Molecular biology of inflammation and sepsis: a primer. Crit Care Med 37(1): 291-304.

5. Taneja R, Parodo J, Jia SH, Kapus A, Rotstein OD, et al. (2004) Delayed neutrophil apoptosis in sepsis is associated with maintenance of mitochondrial transmembrane potential and reduced caspase-9 activity. Crit Care Med 32(7): 1460-1469.

6. Pitrak DL, Tsai HG, Mullane KM, Sutton SH, Stevens $P$ (1996) Accelerated neutrophil apoptosis in the acquired immunodeficiency syndrome. J Clin Invest 98(12): 2714-2719.

7. Salmen S, Montes $H$, Soyano A, Hernández D, Berrueta L (2007) Mechanisms of neutrophil death in human immunodeficiency virus-infected patients: role of reactive oxygen species, caspases and map kinase pathways. Clin Exp Immunol 150(3): 539545.

8. Marick PE (2004) Propofol: therapeutic indications and side effects. Curr Pharm Des 10(29): 36393649 .

9. Kim WH, Jin HS, Ko JS, Hahm TS, Lee SM, et al. (2011) The effect of anesthetic techniques on neutrophil-to-lymphocyte ratio after laparoscopyassisted vaginal hysterectomy. Acta Anaesthesiol Taiwan 49(3): 83-87. 


\section{Anaesthesia \& Critical Care Medicine Journal}

10. Woo JH, Baik HJ, Kim CH, Chung RK, Kim DY, et al. (2015) Effect of Propofol and Desflurane on Immune Cell Populations in Breast Cancer Patients: A Randomized Trial. J Korean Med Sci 30(10): 15031508.

11. Song HK, Jeong DC (2004) The effect of propofol on cytotoxicity and apoptosis of lipopolysaccharidetreated mononuclear cells and lymphocytes. Anesth Analg 98(6): 1724-1728.

12. Tsuchiya M, Asada A, Arita K, Utsumi T, Yoshida T, et al. (2002) Induction and mechanism of apoptotic cell death by propofol in HL-60 cells. Acta Anaesthesiol Scand 46(9): 1068-1074.

13. Utsumi T, Klostergaard J, Akimaru K, Edashige $\mathrm{K}$, Sato EF, et al. (1992) Modulation of TNF-alphapriming and stimulation-dependent superoxide generation in human neutrophils by protein kinase inhibitors. Arch Biochem Biophys 294(1): 271-278.

14. Pithon-Curi TC, Schumacher RI, Freitas JJ, Lagranha C, Newsholme P, et al. (2003) Glutamine delayed spontaneous apoptosis in neutrophil. Am J Physiol Cell Physiol 284(6): C1355-1361.

15. Kishikawa $\mathrm{H}$, Kobayashi $\mathrm{K}$, Takemori $\mathrm{K}$, Okabe $\mathrm{T}$, Ito $\mathrm{K}$, et al. (2008) The effect of dexmedetomidine on human neutrophil apoptosis. Biomed Res 29(4):189-194.
16. Fossati G, Mouding DA, Spiller DG, Moots RJ, White $\mathrm{MRH}$, et al. (2003) The mitochondrial network of human neutrophils: role in chemotaxis, phagocytosis, respiratory burst activation, and commitment to apoptosis. J Immunol 170(4): 19641972.

17. Liu D, Li C, Chen Y, Burnett C, Liu XY, et al. (2004) Nuclear import of proinflammatory transcription factors is required for massive liver apoptosis induced by bacterial lipopolysaccharide. J Biol Chem 279(46): 48434-48442.

18. Watson RW, O’Neill A, Branningan AE, Coffery R, Marshall JC, et al. (1999) Regulation of Fas antibody induced neutrophil apoptosis is both caspase and mitochondrial dependent. FEBS Lett 453(1-2): 6771.

19. Fialkow L, Fochesatto Filho L, Bozzetti MC, Milani AR, Rodrigues Filho EM, et al. (2006) Neutrophil apoptosis: a marker of disease severity in sepsis and sepsis-induced acute respiratory distress syndrome Crit Care 10(6): R155.

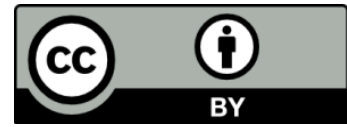

